41 | 2010

Le cheval : monture, nourriture et figure

\title{
L'élevage du cheval au Kirghizstan
}

Horse breeding in Kirghizstan

Amantur Žaparov

Traducteur : Carole Ferret

\section{(2) OpenEdition}

\section{Journals}

Édition électronique

URL : https://journals.openedition.org/emscat/1575

DOI : 10.4000/emscat.1575

ISSN : 2101-0013

Éditeur

Centre d'Etudes Mongoles \& Sibériennes / École Pratique des Hautes Études

Référence électronique

Amantur Žaparov, «L'élevage du cheval au Kirghizstan », Études mongoles et sibériennes,

centrasiatiques et tibétaines [En ligne], 41 | 2010, mis en ligne le 15 avril 2010, consulté le 13 juillet

2021. URL : http://journals.openedition.org/emscat/1575 ; DOI : https://doi.org/10.4000/emscat.1575

Ce document a été généré automatiquement le 13 juillet 2021.

(C) Tous droits réservés 


\title{
L'élevage du cheval au Kirghizstan
}

\author{
Horse breeding in Kirghizstan
}

Amantur Žaparov

Traduction : Carole Ferret

\section{NOTE DE L'AUTEUR}

Cet article est le fruit d'une étude menée au sein d'un groupe de travail international (GDRI) du CNRS intitulé Nomadisme, sociétés et religions dans l'espace turco-mongol et sibérien et soutenu par la Maison des Sciences de l'Homme (FMSH).

\section{Introduction}

1 Pour les Kirghiz, qui passaient naguère plus de temps en selle que sur terre, le cheval est l'un des animaux domestiques les plus proches et les plus prisés. De nos jours, il conserve une place à part parmi le bétail et il n'a pas perdu sa valeur ni économique ni sociale ni culturelle.

2 Plusieurs auteurs se sont penchés sur l'élevage kirghiz du cheval, mais de manière souvent fragmentaire et incomplète, hormis B. B. Akmoldoeva, dont la thèse d'ethnographie (1983) traite exclusivement de cette question, pour la fin du XIX et le début $\mathrm{du} \mathrm{xx}^{\mathrm{e}}$ siècle. En revanche, l'élevage équin du Kirghizstan soviétique et postsoviétique n'a pas fait l'objet d'étude anthropologique ni pluridisciplinaire. Dans cet article, nous nous efforcerons d'éclairer la question par des données rassemblées ces dernières années sur le terrain, principalement dans le nord du pays, en y ajoutant quelques points de comparaison dans le Sud.

3 Nous nous intéresserons à la polyvalence du cheval, aux particularités de son nourrissage, à la politique gouvernementale envers les populations rurales. En effet, au Kirghizstan, le problème des relations entre l'homme, le cheval et l'environnement n'a rien perdu de son actualité. 


\section{Le cheval dans la société traditionnelle}

4 Avant la période soviétique, le statut social des individus était, pour une grande part, déterminé par la quantité de chevaux possédés. Élevé de manière extensive, le cheval était utilisé non seulement pour le transport, mais aussi pour sa viande, son lait, sa peau et ses crins. Ses qualités tenaient à la fois à sa frugalité, à sa capacité à se contenter de vivre du pacage l'année durant, à son exceptionnelle mobilité, si précieuse pour les nomades, enfin à sa poly-fonctionnalité.

5 Le cheval était d'abord un animal de transport, qui permettait de parcourir de grandes distances en un temps réduit. Utilisé sous la selle et le bât, il a facilité la conquête de nouveaux espaces et l'extension du territoire des pâturages (Potapov 2000 ; Žaparov 2002). Pour la nomadisation, le premier animal de bât était le chameau et nombreux étaient ceux qui en possédaient, mais il n'était néanmoins pas rare de voir des chevaux porter des charges.

Chevaux de selle près d'une cascade

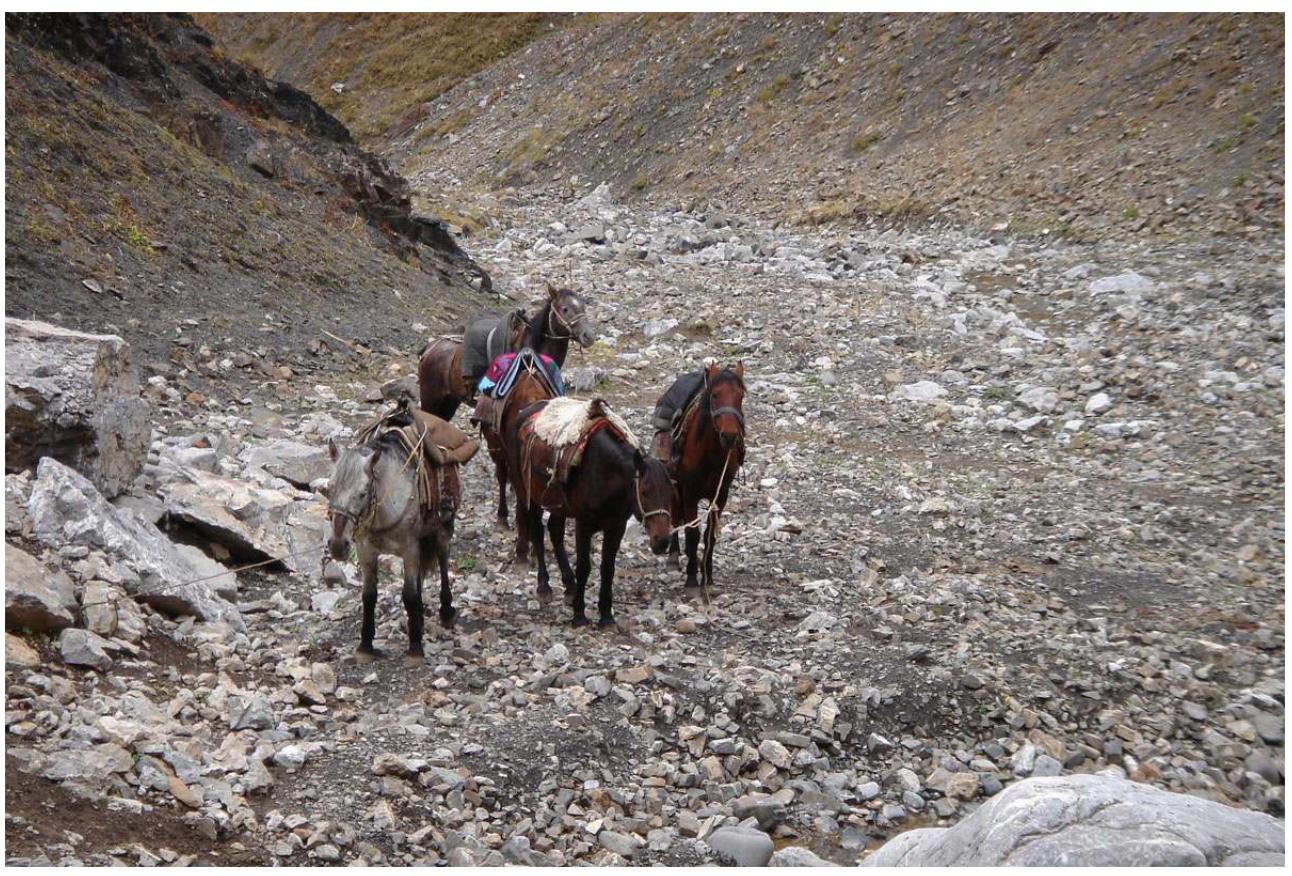

Photographie de A. Japarov. Estive d'At Kulak, arrondissement d'Ak Talin, septembre 2005

Le cheval était employé aux travaux agricoles pour le labour, attelé seul ou par paire à la charrue (soko, buursun), pour la moisson, le battage et le transport des céréales, à l'aide de travois (čijne). Les premières voitures attelées sont apparues dans la deuxième moitié du XIX ${ }^{e}$ siècle, bouleversant le transport dans la région de l'Issyk-kul' (kir. Ysykköl) et la vallée de la čuj. De nos jours, on voit encore, au petit matin les jours de marché, des charrettes aller de l'arrondissement de Džeti-Oguz vers la ville de Karakol ${ }^{1}$. Lors de la crise du début des années 1990, elles étaient utilisées dans de nombreuses régions, par manque de véhicules motorisés ou de carburant, particulièrement en été et à l'automne, pour le transport du foin, des pommes de terre et des autres récoltes. 
7 La chair du cheval fournissait la plus prestigieuse des viandes. Les personnes au statut social élevé dans la société kirghize (bij, manap, datka $a^{2}$ aimaient à se délecter de poulain. Aux premiers froids, un abattage massif (sogum) permettait de faire des provisions de viande pour l'hiver, avec une préférence affichée pour le cheval. Le lait de jument fermenté (kir. kymys, rus. kumys) constituait un élément essentiel de l'alimentation pour de nombreux peuples turco-mongols ( $c f$. Ajtbaev 1963; Kočkunov 2003 ; Jacquesson 2005 ; Ferret 2009). Les crins servaient à fabriquer plusieurs ustensiles ainsi que de grosses cordes (kyl arkan), solides et pratiques, notamment pour fixer les bâts.

Le cheval jouait également un grand rôle dans le cycle de vie des individus. C'était l'espèce de bétail qui comptait le plus dans les compensations matrimoniales (kalyp). De la viande et des abats chevalins ( $\left.k a z y, k a r t a^{3}\right)$ étaient toujours offerts à la belle-famille lors des noces. C'était l'animal sacrifié lors des funérailles (Jacquesson 2007). Des fouilles archéologiques ont révélé que, chez les Turcs anciens (VI-XII siècle), un cheval de selle pouvait être enterré avec le défunt (cf. Tabaldiev 1994). Ce fut aussi parfois le cas chez les Kyrgyz du Iénisséï, l'animal étant censé continuer à servir son maitre dans l'au-delà. S. A. Teplouhov l'a constaté dans des kourganes de pierre datant du vII e siècle, ainsi que pour une partie de ceux des VIII-Xe siècles (cf. Hudjakov 1986, p. 45). Le cheval remplissait plusieurs fonctions dans le rituel funéraire : accompagnement du mort dans la tombe, sacrifice, transport du défunt, viande du festin funéraire (Lipec 1982, p. 214).

Dans la culture des nomades kirghiz, le cheval était également au premier plan pour les jeux et les divertissements. Lors des fêtes saisonnières ou des rites de passage se déroulaient des courses hippiques (at čabyš, alaman bajge, kunan čabyša), des compétitions de lutte à cheval (èniš, oodaryšs) et "d'arrache-bouc » (ulak tartyš, kök börïَ), ainsi que nombre d'autres jeux populaires (cf. Omurzakov \& Saralaev 1981; Simakov 1984; Tynybekov 1993; Ilebaev 2004). Plusieurs d'entre eux ont connu un renouveau avec l'indépendance du pays. Ainsi a été organisé un championnat républicain de kök börü, un jeu qui nécessite non seulement habileté, force et poigne de la part des cavaliers, mais aussi un entraînement spécifique pour les chevaux. Autrefois, les meilleurs coursiers, ainsi que leurs entraîneurs (sajapker), étaient au centre de toutes les attentions. Éleveurs expérimentés, ces derniers connaissaient toutes les ficelles du métier et les subtilités des soins à prodiguer aux chevaux. Acquérant leur renommée par leur savoir-faire et l'accroissant par leurs victoires lors des compétitions, ils étaient en contact avec diverses couches de la population, jouissaient d'un réseau de connaissances étendu et les riches propriétaires recouraient souvent à leurs services.

Le cheval était aussi le compagnon indéfectible du chasseur et sa présence était une condition indispensable au succès de l'activité cynégétique. Avec l'expérience, les montures acquéraient une sensibilité qui leur permettait de détecter la présence de gibier dans les parages et de la signaler à leur maître. Pendant les battues, il était plus facile, avec des chevaux, d'encercler le gibier, de le poursuivre pour l'abattre et enfin de rapporter le butin au campement.

11 Dans sa fonction guerrière, le cheval était sans rival. Lors les incessants conflits avec les voisins ou contre les envahisseurs, il assurait la défense du peuple et du territoire. At attan kijin žat "Le cheval : après lui, couche-toi ", c'est-à-dire " après avoir perdu ton cheval, rends-toi car tu n'es plus en mesure de guerroyer ", dit un proverbe kirghiz. 
Dans l'épopée Manas, le preux réagit violemment lorsque Konurbaj et ses partisans réclament le cheval Maaniker :

At bütköndön ajrylsa S'il est privé de son cheval, Azamat šoru katpajby! L'adversité s'abattra sur le gaillard!

Atyšbaj atyy berdin dep La foudre tombera sur celui qui cède son cheval sans

combattre,

Azabyn artyk tartpajby, Tous les malheurs pleuvront sur sa tête.

Akyrettin künündö Au jour du jugement,

Tozokto Manas žatpajby. Manas peut tomber en enfer.

Maaniker berem degenče Mais céder Maaniker

Manasty öldü deseyči ! Serait, pour Manas, signer son arrêt de mort !

(Manas t. III, 1981, p. 141).

Dans le folklore kirghiz, nombreuses sont les mentions de chevaux qui confèrent aux guerriers une force invincible. Abaz Karymšakov ${ }^{7}$, fin connaisseur des traditions populaires, m'a dit que le cheval était un animal qui avait été créé afin de combattre pour la justice (žylky kazat ülün žaralgan mal). Dans les fréquents conflits auxquels les nomades étaient confrontés, c'était le cheval qui permettait à chaque éleveur de se métamorphoser en guerrier (Hazanov 2008, pp. 16-17). Et c'était à lui qu'il confiait son destin, comme le disait justement B. Soltonoev, auteur qui a vu, pendant la guerre, des gens cacher leurs chevaux préférés dans leur maison, les deux antérieurs liés par des entraves métalliques (kišen) (1993, pp. 186-187).

Sur le rôle militaire du cheval, des données écrites et archéologiques complètent les sources de la littérature orale. Sans son cheval, le nomade cessait d'être un guerrier (Hazanov 2008). Jusqu'à la fin du XIX ${ }^{e}$ siècle, toutes les actions guerrières des Kirghiz pour repousser l'ennemi avaient lieu montées.

Il ne faudrait pas non plus négliger l'utilité du cheval en temps de paix, pour l'organisation du cycle annuel de pacage et la surveillance des troupeaux. Les chevaux permettaient d'exploiter les ressources des pâturages les plus éloignés. Le rayon de pacage aurait été significativement réduit avec des éleveurs à pied, ou même montés sur des bœufs (ögüz) ou des ânes (èšek). À cheval, le gardiennage des troupeaux ovins, bovins ou équins était grandement facilité. Et pour ces derniers, il aurait été impensable de les conduire autrement qu'à cheval. 


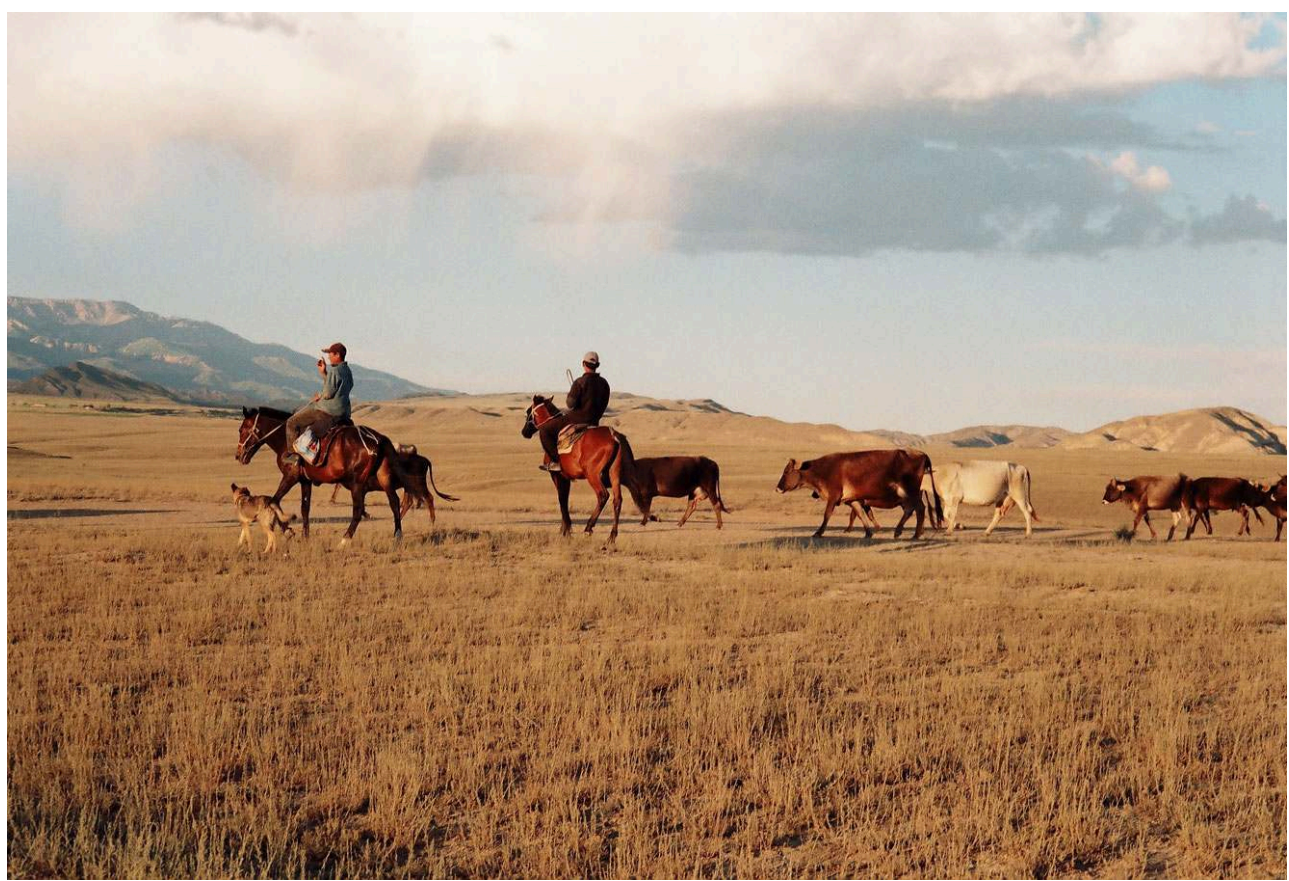

Photographie de A. Japarov. Région de Naryn, septembre 2006

En cas d'enneigement profond ou de verglas sur les pâturages, les éleveurs utilisaient la méthode du pacage successif, y envoyant d'abord les chevaux, seuls capables de dégager la neige et de briser la glace avec leurs sabots (Ajtbaev 1957, p. 50 ; Abramzon 1971, p. 76), puis les autres espèces du bétail. En l'absence d'élevage intensif, c'était là une qualité inestimable du cheptel équin.

Le sabot du cheval kirghiz se distingue par sa petite taille et son exceptionnelle solidité. La corne est si dure que, même non ferré, un cheval kirghiz peut sans dommage parcourir de grandes distances par des routes de montagne caillouteuses et même entièrement pierreuses. Cette qualité, qu'il a manifestement héritée de ses ancêtres sauvages, s'est maintenue grâce à son mode d'élevage semi-sauvage et est une condition indispensable à sa survie. C'est la dureté de son sabot qui lui permet en hiver de [rus.] tebenevat', c'est-à-dire de gratter la neige pour paître (Zujtin \& Vojtjackij 1930, pp. 78-80).

16 C'est pour toutes ces raisons que le cheval représentait, dans le bétail, l'espèce la plus prisée et la plus utile. Il participait à tous les domaines d'activité. Son rôle dans la conquête des nouveaux espaces, dans la diffusion des traits culturels et dans la conduite de la guerre était irremplaçable.

\section{À l'époque soviétique}

17 À l'époque soviétique, les fonctions du cheval se sont un peu restreintes du fait, principalement, de l'utilisation de véhicules motorisés dans les kolkhozes et les sovkhozes. L'élevage du cheval est devenu l'orientation de certaines entreprises collectives spécialisées. Au Kirghizstan, la collectivisation eut lieu dans les années 1930, mais le commencement de la sédentarisation remonte au début $d u x^{e}$ siècle, en particulier parmi les couches les plus pauvres de la population, tandis que les Kirghiz riches ou aisés préféraient toujours demeurer nomades (Saharov 1934, p. 15). 
18 Refusant de céder leurs biens à l'État, nombreux furent les Kirghiz qui opposèrent une résistance farouche à la collectivisation (Džunušaliev 1993, p. 148-168). Dans certaines régions, des éleveurs commirent des actes de violence envers les autorités officielles, d'autres abattirent massivement leur bétail plutôt que de le céder, d'autres enfin fuirent le pays, souvent soutenus par les représentants de l'islam.

19 La sédentarisation massive et forcée eut lieu à partir de 1931. Ceux qui étaient considérés comme des koulaks furent exilés : non seulement des éleveurs riches, mais aussi d'autres qui subvenaient tout juste à leurs besoins. Le bétail de ces "riches " expropriés constitua l'essentiel du cheptel collectivisé. Par exemple, sur le territoire de l'actuel arrondissement d'Ak-Talaa, dans la région de Naryn, les quatre-vingt-cinq chevaux, dix chameaux, quinze vaches et trois cent soixante-quinze moutons d'un certain Musakožo permirent de former l'artel agricole Kyzyl Èmgek « Travail rouge ", composé de quarante foyers (Ak-Talaa 1996, p. 12). Présentée comme une condition nécessaire au développement culturel du peuple et à la reconstruction de l'économie, la sédentarisation était surtout motivée, en réalité, par la volonté d'atteindre l'autosuffisance céréalière.

20 Dans les entreprises collectives, les bergers disposaient de chevaux de selle pour garder et déplacer les troupeaux. Les gardiens de troupeaux de chevaux étaient nettement moins nombreux. Beaucoup exerçaient leur profession de père en fils, les plus méritants étaient récompensés par des décorations officielles. En outre, la république comptait quelques haras.

21 Dans la sphère privée, le nombre de têtes de bétail que chaque kolkhozien ou chaque sovkhozien était autorisé à posséder individuellement connut des variations historiques et géographiques mais il a toujours été strictement limité. Par exemple, dans l'arrondissement Tonskij de la région de l'Issyk-kul', chaque famille, ou plus exactement chaque foyer (tütün), la plus petite unité économique, pouvait avoir, dans les années 1970, une jument avec un poulain, une vache avec un veau et vingt moutons. Les bêtes supplémentaires étaient confisquées par les commissions des kolkhozes et des sovkhozes lors de leurs tournées d'inspection.

Ces commissions étaient composées du personnel dirigeant des fermes collectives et de représentants des sel'sovet « soviet rural » (souvent son secrétaire). Toutes les familles ne souhaitaient pas céder leur poulain ou leur veau, même contre rétribution, mais la loi autorisait à les leur confisquer. Ces confiscations permettaient également de réaliser le plan en matière de livraison de viande à l'État.

23 Les gens parvenaient néanmoins à ruser pour détenir plus de bétail qu'il n'était permis. Ils dissimulaient leurs bêtes. L'auteur de ces lignes, qui est né et a grandi au village de Kara Koo, dans la région de l'Issyk-kul', a souvent été témoin de telles scènes dans la première moitié des années 1970. À Togolok Moldo dans l'arrondissement d'Ak-Talaa, à Korkul dans celui d'Alaj et à Songköl dans celui de Kočkor, des informateurs m'ont raconté que les propriétaires s'efforçaient de conserver plus de chevaux qu'ils n'étaient autorisés à le faire. Il arrivait parfois que des animaux, cachés dans un bâtiment, révèlent eux-mêmes leur présence à la commission, dénonçant leur maître par un hennissement ou autre bruit semblable. Les gardiens de troupeaux étaient privilégiés en la matière, car ils pouvaient garder leurs bêtes dans le troupeau collectif et même obtenir de la nourriture supplémentaire aux frais du kolkhoze ou du sovkhoze. En cas d'inspection, ils séparaient leurs bêtes du troupeau et les cachaient dans un endroit 
isolé, comme une petite gorge. De cette manière, certains parvenaient à conserver quelques chevaux supplémentaires.

\section{Transformations récentes}

24

$\mathrm{Au}$ moment de la chute de l'URSS, le secteur de l'élevage était déjà en crise. Après l'indépendance, le gouvernement mena une politique de réforme agraire qui se manifesta par la privatisation des terres cultivées et du bétail et bouleversa l'organisation économique et sociale.

Lors de la privatisation, les pâtres de certaines espèces furent favorisés par rapport au reste de la population pour la distribution du bétail. Ainsi, les gardiens de troupeaux de chevaux reçurent davantage de chevaux. Ce fut le cas de Kubanyčbek Mambetov, par exemple, gardien de troupeaux de chevaux dans la région d'Ak Talaa. Son père, qui exerçait la même profession, avait été récompensé du titre de «Héros du travail socialiste " pour les excellents résultats qu'il avait obtenus. C'est pour cette raison que son fils reçut six chevaux lors de la privatisation du bétail, alors que d'autres n'en eurent aucun. Aujourd'hui, dans les régions de Naryn ou de l'Issyk-kul', certains anciens gardiens de troupeaux de chevaux possèdent quarante, cinquante chevaux, voire plus. C'est non seulement l'appât du gain, mais aussi leur connaissance et leur amour des chevaux qui leur ont permis de constituer de tels troupeaux.

Dans les premières années de l'indépendance, les anciens membres des kolkhozes et des sovkhozes n'étaient pas prêts à entretenir convenablement le bétail. Le manque de fourrage, l'absence de soins vétérinaires et de souci zootechnique, la mauvaise qualité des pâtures ont provoqué d'immenses pertes dans le cheptel. Entre 1991 et 1997, le nombre de bovins a baissé de $23 \%$ et celui du petit bétail (ovin et caprin) de $62 \%$ (Gusev 2000, p. 5).

27 La solvabilité de la population était très faible, les éleveurs ont vendu massivement leurs bêtes et le bétail s'est fortement dévalué. Un de nos informateurs, qui faisait paître ses bêtes sur le pâturage de Kyzart, nous a raconté qu'au début des années 1990, tout le monde s'est vu contraint de vendre ou d'échanger du bétail. Pour un sac de farine, quelques paquets de thé ou deux-trois bouteilles de vodka, on donnait un mouton adulte. La population ne pouvait se procurer des médicaments ou des biens de première nécessité qu'en troquant ses bêtes. Le bétail a alors perdu de sa valeur pécuniaire ${ }^{9}$ mais aussi sociale. Le prestige de la profession d'éleveur (de moutons, de chevaux, de yaks) s'est évanoui du jour au lendemain.

Beaucoup, néanmoins, continuaient à apprécier le cheval, même en ces temps de crise et pour eux il gardait la place à part qu'il a toujours eue dans la vie économique et culturelle des Kirghizes. Sa frugalité a aussi joué en sa faveur car il peut se contenter de paître en s'éloignant des habitations. La baisse du cheptel équin (-13 000 têtes) dans la première moitié des années 1990 est moins liée à des pertes subies, dues à l'inanition et aux maladies, qu'à des abattages délibérés, pour célébrer différentes fêtes, aux niveaux familial, local et gouvernemental. En effet, la viande de cheval reste de mise pour les funérailles et les jubilées.

Par ailleurs, les loups (karyškyr) sont une véritable plaie pour le bétail au Kirghizstan, d'autant que la grande majorité des éleveurs n'a pas de fusil - souvent, la police le leur a confisqué car ils n'avaient pas de permis de détention d'armes. Mes informateurs se 
plaignent fréquemment de la bureaucratie et de la sophistication du système des permis. À la fin des années 1990, le gouvernement payait environ 1000 soms pour la peau d'un loup abattu. Mais cela donnait souvent lieu à des litiges. En l'absence de permis, le fusil était aussitôt confisqué. À l'époque soviétique, on distribuait des fusils à canon plat aux éleveurs, pour qu'ils puissent protéger leur troupeau. Et quand le nombre de loups dépassait une certaine norme, les chasseurs organisaient de grandes battues. Lors d'une expédition ethnographique menée dans les années 1998-2000 sur l'état actuel de l'élevage ${ }^{10}$, les éleveurs se sont plaints de ce que les loups s'aventuraient à présent jusque dans les villages, sans que les autorités officielles, ni locales ni gouvernementales ne prennent la moindre mesure.

Le vol des chevaux était de longue date une autre plaie endémique. Les propriétaires voyaient parfois plusieurs têtes de bétail disparaître d'un coup et les voleurs revendaient les chevaux volés ou leur viande afin, pour certains, d'éponger leurs dettes. Ces dernières étaient amplifiées par les taux d'intérêt élevés auxquels des privés prêtaient alors de l'argent, comme en témoignaient les media, ainsi que nos informateurs.

31 À partir de 2000, la situation a commencé à s'améliorer, les gens ont pu de nouveau se consacrer à l'élevage et le cheptel a crû. La République, qui comptait 308100 chevaux en 1996, en avait 354400 en 2002 et 384000 en 2006. En outre, la population rurale préfère le gros bétail (bovin et équin), plus rentable dans les nouvelles conditions du marché. Peu exigeants, les chevaux peuvent paître même lors des plus grands froids et leur prix n'est jamais tombé très bas. Ces dernières années, un cheval adulte et bien nourri se vend entre 1000 et 1500 dollars sur les marchés. Depuis 1995, des gens viennent du Kazakhstan pour en acheter, ce qui a stimulé l'élevage équin. Par ailleurs, les alpages, qui n'étaient plus foulés par les sabots du bétail, se sont régénérés. Et les éleveurs kirghiz, ces descendants de nomades, se sont remis du choc qu'ont représenté pour eux la fin de l'Union soviétique et la crise profonde qui avait suivi. Ils ont recommencé à exploiter les hauts pâturages, riches en herbes juteuses. Désormais, des nuées de bétail en redescendent à l'automne.

Il fallait des chevaux de selle pour conduire ces troupeaux sur les collines et les pentes des montagnes. La demande en kumys ne cessait de croitre. Les cordes d'attache pour les poulains (žele) se mirent à s'allonger sur les estives. Après quelques années de crise, on revoyait des troupeaux de chevaux (üjür) près des iourtes (boz üj) à l'heure de la traite alors que, jusqu'à la fin des années 1990, seuls quelques éleveurs isolés fréquentaient les alpages. 


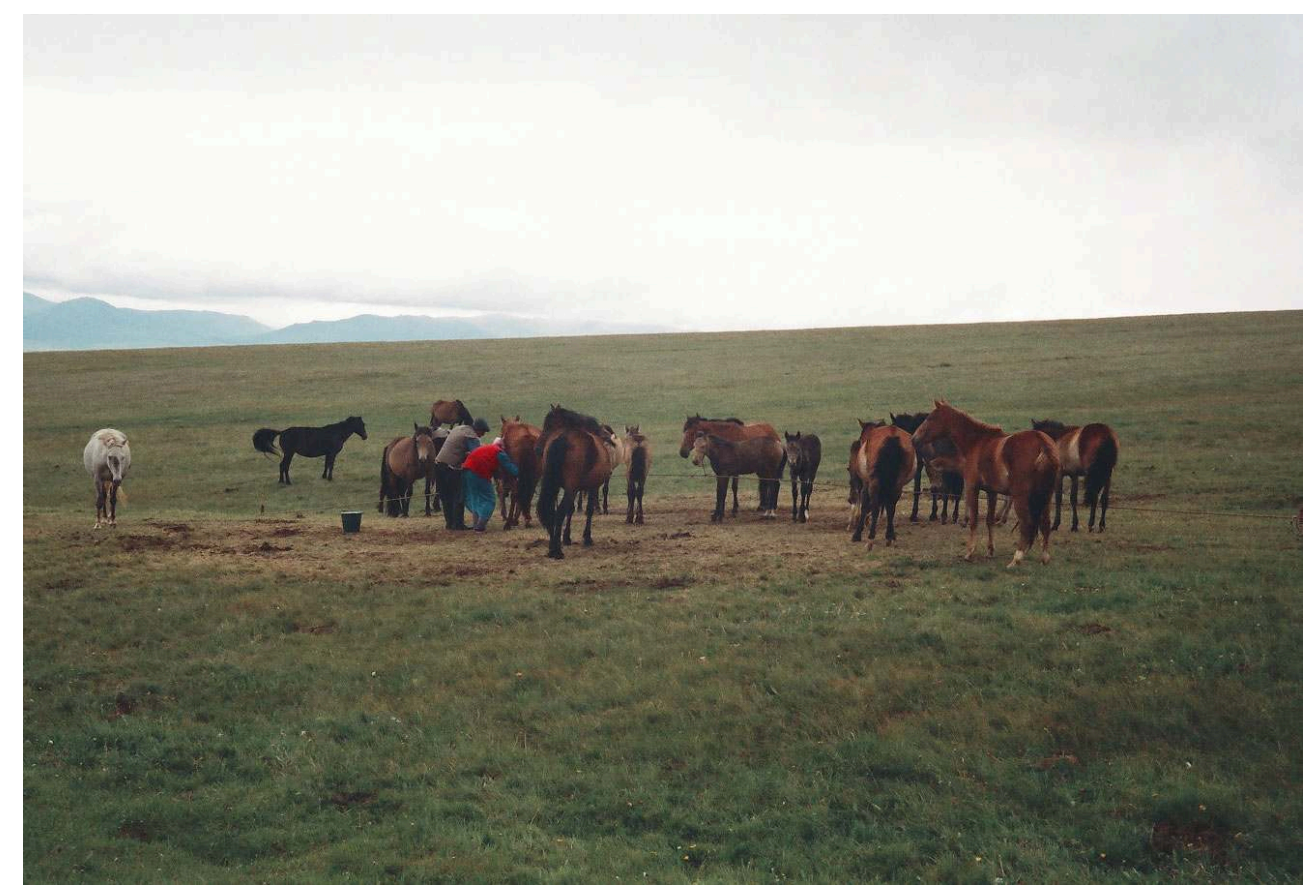

Photographie de A. Japarov. Tepši, Son-Köl, juillet 2005

Après l'indépendance, certaines personnes, fonctionnaires ou entrepreneurs prospères, se sont mises à élever des chevaux de race. Ainsi le célèbre politicien F. Kulov, ancien akim "chef de l'administration du rajon " de l'arrondissement Čymyrbaev, ou I. Omurkukov, député au parlement, ont pour hobby l'élevage des chevaux - bien qu'il soit difficile d'évaluer leur influence en ce domaine. Tandis que les haras d'État traversaient de graves difficultés, des haras privés et des coopératives se sont lancés dans l'aventure. Le haras Ajkol, dans l'arrondissement Tonskij de la région de l'Issykkul' par exemple, bénéficie d'une collaboration avec des spécialistes de l'université agricole d'État pour élever des chevaux locaux, kirghiz, améliorés ainsi que quelques pur-sang, qui participent à des grandes courses au Kirghizstan et au Kazakhstan, grâce à l'activisme de son propriétaire, chaud partisan des jeux équestres et des courses hippiques.

Le projet Kyrgyz Aty, promu par une Française, Jacqueline Ripart, vise à sauvegarder et à développer la race locale. Dans cet objectif, plusieurs festivals At čabyš ont été organisés sur la rive sud de l'Issyk-kul', comprenant des courses, des jeux équestres et des concerts. En outre, cette organisation non gouvernementale mène une propagande active auprès de la population rurale, afin de lui faire prendre conscience de la valeur inestimable du cheval kirghiz dans ce pays de montagnes.

Le renouveau des jeux et divertissements équestres après l'indépendance a permis d'attirer l'attention sur l'élevage équin. Des courses et des jeux d'« arrache-bouc » ont été organisés de plus en plus fréquemment, aux niveaux local et national, parfois avec la participation de cavaliers des républiques voisines, voire de l'Altaï et de Khakassie (Fédération de Russie). Dans certains arrondissements du sud du pays (Alaj, Lejlek, entre autres), l'ulak tartyš se pratiquait déjà souvent à la période soviétique, généralement lors des fêtes familiales. En 1988, j'ai assisté pendant près de sept heures à l'un de ces jeux au village Kara Bulak de l'arrondissement Lejlek, avec des 
participants venus du Tadjikistan voisin, à l'occasion de la circoncision (sünnötkö oturguzuu) du fils d'une personnalité influente du village.

Dans le Sud, on mange moins de chevaux, non que ceux-ci soient moins nombreux, mais à cause d'une certaine réticence envers l'hippophagie liée à l'islam. Dans les arrondissements de Karasuu et d'Aravan, on consommait habituellement très peu de cheval mais, depuis cinq ou six ans, on en abat de plus en plus pour les fêtes ou les funérailles. Là-bas, les chevaux demeurent néanmoins avant tout des animaux de selle ou de bât, utilisés pour le transport sur les routes de montagnes escarpées menant aux alpages.

S'adaptant aux nouvelles conditions économiques, les éleveurs se sont mis, depuis quelques années, à louer des chevaux aux touristes étrangers. Saisissant l'opportunité du développement touristique du pays, quelques familles s'efforcent de mieux soigner leurs chevaux et de disposer d'une sellerie correcte afin de satisfaire leurs clients, y compris dans leurs exigences esthétiques. Ils savent parfaitement que le cheval est à même de leur procurer une source de revenus supplémentaires sur les alpages.

Ces derniers temps, le lait de jument est devenu populaire comme jamais. Des malades et des gens soucieux de leur santé le boivent frais (bè̀nin sütü) ou légèrement fermenté (saamal). On dit que, pour conserver toutes ses propriétés, le lait cru doit être avalé avant même d'avoir eu le temps de refroidir. Certains consomment exclusivement le lait d'une jument particulière. D'autres préfèrent le saamal pour les soins ou la prophylaxie. La hausse de la demande a fait grimper le prix de ces produits, surtout dans les montagnes proches de la capitale, où le litre vaut cent cinquante soms (environ trois euros). Les pensionnaires de la maison de repos des sources chaudes d'Ysyk Ata peuvent en commander, ainsi que les habitants de Bichkek, mais on estime qu'il est préférable de boire du lait de jument sur le lieu même de production, en contemplant les juments et les poulains à l'attache.

39 Autrefois, les Kirghiz rechignaient à faire commerce de produits laitiers : akty satpajt "les laitages, ça ne se vend pas ", disait-on. Cette réticence a pratiquement disparu aujourd'hui. Des entreprises locales spécialisées dans la production de boissons rafraîchissantes achètent beaucoup de lait. À Tepši par exemple, près du lac de montagne Son-Köl, une base de la firme Šoro achète du lait de jument aux éleveurs transhumants afin de le transformer en kumys. Elle a fixé un prix d'achat assez bas, qu'elle justifie en arguant de l'éloignement, du mauvais état des routes et des dépenses liées au transport. Les éleveurs lui vendent néanmoins volontiers le lait de leurs juments, car cela leur assure une source d'argent liquide. Des petits commerçants font concurrence à cette firme, en achetant aussi du kumys aux éleveurs, mais leur volume d'achat est plus réduit et plus aléatoire.

40 Il existe ainsi une série de facteurs qui favorisent actuellement l'élevage du cheval dans le pays. Ce secteur a fait preuve de sa faculté d'adaptation aux nouvelles conditions économiques et à la globalisation. Au niveau gouvernemental, quelques mesures ont été prises pour le développement de l'élevage équin au Kirghizstan. Un programme d'État pour les années 2003-2010, approuvé par un arrêté du gouvernement en date du 22 mai 2003, fixe comme objectif principal à moyen terme du développement de la filière équine, une remonte suffisante en chevaux de qualité (reproducteurs, bouchers, laitiers, de travail et de sport) afin de satisfaire aux besoins des ménages et des entreprises agricoles, sportives et autres, publiques et privées, en vertu de la grande rentabilité de cet élevage (Gosudarstvennaja programma 2003 ; Žaparov 2007). 


\section{Nourrissage et soins des chevaux}

À présent, l'attitude des propriétaires envers leurs chevaux dépend grandement de la destination de ces derniers (reproduction, alimentation, travail, compétition, tourisme). Les uns prêtent une attention accrue au moindre détail de leur comportement et veillent scrupuleusement à leur bien-être, tandis que les autres sont moins regardants. Le prix des animaux entre également en ligne de compte. Un bon ambleur ou un coursier capable de remporter des prix lors des compétitions vaut bien plus cher qu'un simple cheval de travail ${ }^{11}$. Ceux-là exigent des soins plus assidus pour leur alimentation, leur entraînement (taptoo) et leur surveillance.

Des chevaux paissent dans un col de montagne

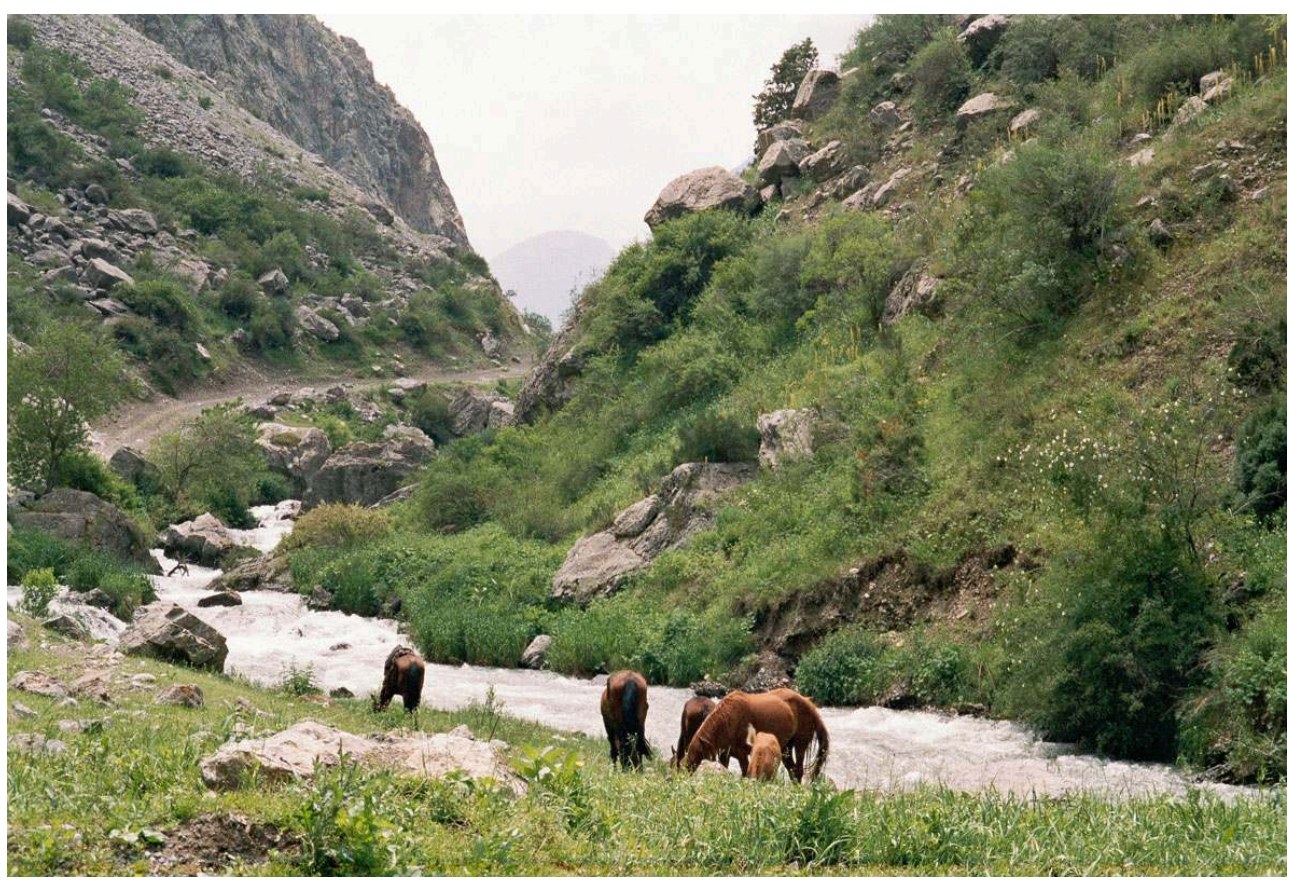

Photographie de A. Japarov. Apšyr Ata, arrondissement de Nookat, juin 2003

Les chevaux passent généralement la saison chaude dehors. Les chevaux de selle sont entravés (tušajt) sur les pâtures afin qu'ils ne puissent pas partir au loin, ou bien attachés par une longue corde à un piquet (kazyk) une fois dessellés (žajdaktalat). On procède de même durant la journée, quand on sait qu'on aura encore besoin de leurs services. Après un parcours à allure vive ou le transport d'une charge, les chevaux sont toujours mis à l'attache (suutuu), comme chez l'ensemble des pasteurs de Sibérie et d'Asie centrale (Ferret 1998; Ferret 2006, pp. 624-643). Ce sont les adultes ou les enfants, sauf les plus jeunes, qui s'en occupent et veillent à ce que le cheval en sueur ne prenne pas froid, aussi lui mettent-ils souvent un tapis (terdik) sur le dos, attaché avec une sangle (basmajyl). S'il a travaillé dur, on l'attache court assez longtemps afin qu'il reprenne sa respiration, refroidisse et se repose. Si le cavalier est arrivé au crépuscule, il doit penser à se lever au milieu de la nuit pour libérer le cheval, le nourrir, l'abreuver et laisser paître. Sans nécessiter un gros travail, ces gestes manifestent néanmoins l'attention portée par son maître à l'animal. 

(üjür žylky) dirigé par un étalon (ajgyr) ne s'éloignent guère de l'endroit où sont attachés leurs poulains, où on les amène cinq à six fois par jour pour la traite; mais, la nuit, les poulains (kulun) sont remis en liberté et tous, juments et poulains, peuvent monter sur les alpages (tör). Tôt le matin, un membre de la famille va les chercher à cheval. Généralement, il les regroupe sans difficulté car le troupeau, déjà habitué, se dirige de lui-même vers la corde d'attache (žele). Sur le campement, la femme et les enfants attrapent les poulains, les attachent et traient les juments. troupeau de chevaux " est généralement de bien plus grande taille que les rus. kosjak "troupeau, manade ", dont il est formé et qui sont chacun constitués d'un étalon (ajgyr) et de dix à quinze poulinières (bèè) (Ferret 2006, pp. 186-187). À la saison chaude, chaque kosjak paisse séparément et en liberté (Sydykov 1992). Sur les estives, on les voit ainsi aller et venir près des crêtes des montagnes. Les éleveurs contrôlent simplement leur présence un jour sur deux. Ce qu'ils craignent avant tout, ce sont les attaques des loups ainsi que les vols, surtout en période de crise économique. Il arrive aussi que le troupeau change de pâture et parte au loin. Les bergers peuvent alors fournir des renseignements aux éleveurs de chevaux sur l'emplacement de leurs bêtes. Tous les gens qui ont l'habitude de participer à la transhumance savent reconnaitre la plupart les chevaux du village. Les éleveurs s'aident donc d'échanges verbaux pour contrôler les mouvements du troupeau.

À la saison froide, beaucoup de chevaux sont gardés en semi-stabulation. D'autres restent en pacage libre, sur des pâtures peu éloignées, en fonction des conditions naturelles et climatiques locales. Là où l'épaisseur de la couche de neige est peu importante, les chevaux peuvent paître loin du village. Dans le cas contraire, on les dirige vers des lieux où ils parviennent à gratter la neige avec leurs sabots pour atteindre l'herbe. Durant l'hiver, on leur distribue une alimentation complémentaire : du foin (čöp) fauché à la fin de l'été sur des prairies naturelles et artificielles (luzerne, sainfoin).

Les chevaux de travail utilisés sous la selle ou attelés font l'objet de soins plus attentifs. Leurs dépenses énergétiques étant supérieures, on leur donne aussi des céréales (žem): orge, avoine, maïs ou granulés dans une musette (žem baštyk).

De même, on s'occupe activement des chevaux destinés à être abattus pour des fêtes ou des funérailles. Ils sont mis à l'embouche, bien soignés, protégés des voleurs et abrités du mauvais œil, afin de bien engraisser avant d'être vendus sur les marchés à bestiaux.

En hiver, les chevaux en stabulation ou en semi-stabulation sont placés pendant la nuit dans des abris couverts (bastyrma), dont l'un des côtés - le moins venteux - reste ouvert et dont le toit peut être surmonté d'une meule de foin. Vaches et moutons sont placés à côté, dans un autre box. Dans les fermes où l'élevage équin est primordial, les conditions d'hivernage sont plus confortables pour les chevaux.

En hiver, les soins sont plus assidus. Le propriétaire doit veiller à abreuver ses chevaux, soit en les emmenant à l'abreuvoir, soit en leur apportant de l'eau dans un seau, les alimenter en fonction de leur travail, nettoyer le fumier. Certains confectionnent des litières avec du crottin séché (astyn kurgaktajt) pour que les animaux ne se couchent pas sur un sol humide. Ce sont généralement le maître de maison et ses fils qui s'occupent 
des chevaux, rarement sa femme et ses filles car la division sexuelle du travail est assez stricte et la majeure partie des tâches liées à l'élevage échoit aux hommes.

Conformément à la culture équestre kirghize qui s'est développée durant des siècles, les chevaux sont, en dehors de quelques exceptions, bien traités. Il est très mal vu de frapper un cheval sur la tête ou de le faire travailler jusqu'à l'éreinter. Le harnais de selle et de trait doit être en bon état, dans la mesure du possible, et bien placé, pour ne pas provoquer de plaie ni de gonfle (žoor) sur le dos.

51 Dans le nord du pays, deux types de ferrure sont employés en fonction du terrain. Certains fers sont munis de trois crampons pour éviter les glissades en terrain verglacé et pentu mais, selon mes informateurs, ils ne sont pas toujours pratiques d'utilisation et des fers sans crampons peuvent leur être préférés. Pour le ferrage (at takaloo), on fait généralement appel à un spécialiste du village, sauf si un membre de la famille est capable de s'en charger. Il s'effectue à froid, avec des fers achetés sur les marchés aux bestiaux.

52 Pour soigner les maladies équines, les Kirghiz ont encore recours aux remèdes traditionnels, mais la majorité fait néanmoins confiance aux vétérinaires et aux zootechniciens spécialisés. Étant donné que beaucoup d'éleveurs ont subi de grandes pertes ces dernières années, ils prêtent désormais plus attention à la prophylaxie. En dépit du coût que cela implique, ils se sont remis à vacciner leurs bêtes et à leur donner des médicaments comme on le faisait dans les sovkhozes et les kolkhozes. Les propriétaires ont compris l'importance de la vaccination afin de sauvegarder leur cheptel et donc leur bien. Y contribuent non seulement l'émergence d'une nouvelle mentalité, issue de la privatisation, mais aussi l'action de certains vétérinaires privés auquel le ministère de l'agriculture donne, comme à ceux de l'État, le droit d'exercer dans les villages.

\section{Conclusion}

53 Au Kirghizstan, le cheval est proche de l'homme par les nombreuses fonctions qu'il remplit comme par les soins que ce dernier lui prodigue. Une relation de confiance s'établit entre ces deux êtres. Pour son maître, la mort d'un cheval représente plus qu'une perte économique, un choc émotionnel. P. P. Semenov-Tjan-Šanskij relate ainsi un épisode de son voyage dans le pays, au milieu du XIX siècle :

À un endroit, un de nos chevaux est mort en tombant dans un ravin. Il a fallu remonter sa charge et la partager entre les trois chevaux de réserve. Le Kirghiz auquel ce cheval appartenait l'étreignit et pleura comme s'il avait perdu un ami. Il coupa un morceau de son oreille et de sa queue et il les emporta avec lui (1958, p. 184).

Ce geste, peut-être étrange aux yeux des occidentaux, manifestait en réalité la sincérité de son chagrin.

L'homme et le cheval - surtout le cheval de selle, qui accompagne partout son maitre deviennent des amis inséparables. Les enfants apprennent à s'occuper des chevaux en soignant la monture de leur père. Le respect de l'animal représente une étape importante du processus de socialisation. Et le cheval, en retour, est sensible à l'attention qu'on lui porte. 

cheval. C'est cet animal qui, en nourrissant l'homme, en le transportant, en favorisant la circulation de l'information, en l'aidant à combattre ses ennemis, a permis le développement du nomadisme pastoral. Et aujourd'hui, en dépit des difficultés survenues lors de la privatisation du bétail, la filière équine continue à faire vivre une partie de la population.

\section{BIBLIOGRAPHIE}

Abramzon, S. M.

1971 Kirgizy i ih ètnogenetičeskie $i$ istoriko-kul'turnye svjazi (Leningrad, Nauka).

Ajtbaev, M. T.

1957 Istoriko-kul'turnye svjazi kirgizskogo i russkogo narodov (Frunze, Izd. AN Kirg. SSR).

1963 Pišča kirgizov (konec XIX - načalo XX v.), Izvestija Akademii nauk Kirg. SSR, serija obščestvennye nauki, t. 5, vyp. 1 (Istorija), pp. 19-23.

Akmoldoeva, B. B.

1983 Konevodstvo v sisteme tradicionnogo hozjajstva kirgizov (konec XIX - načalo XX v.) (Moscou, Dissertacija na soiskanie učenoj stepeni kandidata istoričeskih nauk - ètnografija).

Ak-Talaa - kuttun žergesi 1996 (Bichkek, s. ed.).

Brusina, 0 .

2001 Slavjane v Srednej Azii (Moscou, Nauka).

Džunušaliev, Dž.

1993 Vremja sozidanija i tragedij (Bichkek, Ilim).

Ferret, C.

1998 [Ferre K.] O vystojke lošadej u tjurko-mongol'skih narodov [Sur l'attache des chevaux chez les peuples turco-mongols], Lavrovskie (sredneaziatsko-kavkazskie) čtenija, 1996-1997 gg. Kratkoe soderžanie dokladov (Saint-Pétersbourg), pp. 57-59.

2006 Techniques iakoutes aux confins de la civilisation altaïque du cheval. Contribution à une anthropologie de l'action (Paris, EHESS. Thèse de doctorat en anthropologie sociale et ethnologie) 3 vol.

2009 Une civilisation du cheval. Ses usages de la steppe à la taïga, (Paris, Belin).

Gosudarstvennaja programma razvitija konevodstva $v$ Kyrgyzskoj Respublike na 2003-2010 2003 en ligne http://www.government.gov.kg

Gusev, K. B.

2000 Èkonomika Kyrgyzstana v preddverii xx veka, Reforma, 2, p. 5.

Hazanov, A. M.

2008 Kočevniki i mirovoj istoričeskij process, in Vklad kočevnikov v razvitie mirovoj civilizacii (Almaty, Daik-Press), pp. 16-17.

Études mongoles et sibériennes, centrasiatiques et tibétaines, 41 | 2010 
Hudjakov, Ju. S.

1986 Kyrgyzy na Enisee (Novossibirsk, Novosibirskij gosudarstvennyj universitet imeni Leninskogo komsomola).

Ilebaev, A. K.

2004 Kirgizkie igry i razvlečenija (Bichkek, Ilim).

Jacquesson, S. [Žakson, S.]

2005 Kakovy že vse-taki dostoinstva kumysa : èkologičeskie ili social'nye, in Nasledie material'noj $i$ duhovnoj kul'tury (Bichkek, Ilim), pp. 77-86.

2007 Le cheval dans le rituel funéraire kïrgïz. Variations sur le thème du sacrifice, Journal asiatique, 295 (2), pp. 383-414.

Kočkunov, A. S.

2003 Tradicionnaja pišča kyrgyzov (nekotorye voprosy tipologii), Bilim žana tarbija, 2, p. 7-14.

Lipec, R. S.

1982 Otraženie pogrebal'nogo obrjada v tjurko-mongol'skom èpose, in Obrjady i obrjadovyj fol'klor (Moscou, Nauka).

Manas

1978-1982 (Frunze, Kyrgystan, version de l'épopée par S. Orozbak), 3 t.

Omurzakov D. \& M. Saralaev

1981 Kirgizskie narodnye vidy sporta i narodnye igry (Frunze, Kyrgyzstan).

Potapov, L. P.

2000 Kul'tura altajcev - naslednica drevnej civilizacii kočevnikov Central'noj Azii, Ètnografičeskoe obozrenie, 5, pp. 31-41.

Saharov, M. G.

1934 Osedanie kočevyh i polukočevyh hozjajstv Kirgizii (Moscou, Trudy NII zhivotnovodstva Kirg.

ASSR).

Semenov-Tjan-Šanskij, P. P.

1958 Putešestvie v Tjan'-Šan' (Moscou, Gosudarstvennoe izdatel'stvo geografičeskaja literatura).

Simakov, G. N.

1984 Obščestvennye funkcii kirgizskih narodnyh razvlečenij v konce XIX - načale XX v. (Leningrad,

Nauka).

Soltonoev, B.

1993 Kyzyl kyrgyz taryhy (Bichkek, Učkun), t. 1.

Sydykov, A.

1992 Organizacija vypasa v kočevoj gruppe, in Z. Kurmanov, V. M. Ploskih, S. Begaliev,

Č. Nogojbaeva \& Z. F. Sorokina (ed.), Abdykerim Sydykov-nacional'nyj lider (Bichkek, Kyrgyzstan), pp. 124-130.

Tabaldiev, K. ̌̌.

1994 Èvoljucija pogrebal'nogo obrjada kočevnikov Vnutrennogo Tjan'-Šanja v srednie veka (Novossibirsk, Avtoreferat dissertacii na soiskanie učenoj stepeni kandidata istoričeskih nauk), 22 p.

Tynybekov, A.

1993 Kyrgyzdyn uluttuk ojundary, in Kyrgyzdar (Bichkek, Kyrgyzstan), pp. 530-584.

Žaparov, A. Z.

2002 Tradicionnoe skotovodstvo i social'naja organizacija u kyrgyzov (Bichkek, Avtoreferat dissertacii 
na soiskanie učenoj stepeni kandidata istoričeskih nauk).

2007 Sovremennye kočevniki vysokogornogo Naryna i rynok, in Mir kočevyh civilizacij : istorija $i$ sovremennost' (Čita, Aginskoe, Materialy meždunarodnoj naučnoj konferencii. 10-12 oktjabrja 2007 goda), pp. 313-318.

Zujtin, K.K. \& B. P. Vojtjackij

1930 Lošad' kočevogo naselenija Kirgizii in Materialy komissii èkspedicionnyh issledovanij, vyp. 22, p. 5-155.

\section{NOTES}

1. On trouve le dessin d'une brička, voiture légère utilisée par des colons slaves au nord du lac Issyk-kul', dans le livre d'O. Brusina (2001, p. 39, ill. 4).

2. Jusqu'au XVIII ${ }^{\mathrm{e}}$ siècle, les tribus étaient dirigées par des bij puis, par la suite, la classe dirigeante fut désignée par un autre terme (manap), tandis que les bij se voyaient confier un rôle judiciaire, pour la résolution des conflits selon le droit coutumier. Le titre de datka était l'un des plus élevés dans le khanat de Kokand.

3. Le kazy désigne à la fois la graisse abdominale et costale ainsi qu'un saucisson fabriqué avec la viande des côtes. Le karta correspond au rectum gras, également très apprécié.

4. At čabyš : course de chevaux. Kunan čabyš : course des poulains de deux ans. Alaman bajge : la plus importante des courses hippiques, à laquelle participaient les meilleurs chevaux. Sur les courses hippiques en Mongolie et l'entraînement des chevaux de courses au Kazakhstan, voir les contributions de G. Lacaze, C. Ferret et A. Toqtabaev dans le présent recueil.

5. Èyiš et oodaryš sont les deux noms d'un même jeu où deux cavaliers s'affrontent en tentant de se désarçonner.

6. Ulak tartyš «arrache-bouc ", kök börü, littéralement «loup bleu »: jeu collectif où des cavaliers doivent saisir sur le sol le cadavre d'une chèvre ou d'un veau et le jeter en un lieu déterminé.

7. Informateur né en 1902, arrondissement d'Ak Talin dans la région de Naryn.

8. Toutes les statistiques sur le cheptel doivent être considérées avec une certaine prudence, mais d'après la FAO, les chiffres sont les suivants :

\begin{tabular}{|l|l|l|l|l|l|l|}
\hline & 1992 & 1995 & 1998 & 2001 & 2004 & 2007 \\
\hline chevaux & 320500 & 299000 & 325400 & 353900 & 340505 & 347526 \\
\hline chèvres & 300000 & 152000 & 380000 & 601429 & 769548 & 849873 \\
\hline moutons & 9225000 & 4924400 & $(3424900)$ & 3197760 & 2883920 & 3197076 \\
\hline chameaux & $(500)$ & $(500)$ & $(450)$ & $(300)$ & 434 & 291 \\
\hline bovins & 1190000 & 920100 & 884800 & 947021 & 1004363 & 1116733 \\
\hline
\end{tabular}

Évolution du cheptel au Kirghizstan depuis l'indépendance (en têtes de bétail. Les parenthèses signalent des estimations ou des chiffres non officiels). Source http://faostat.fao.org

A noter, comme dans d'autres pays de la zone, un boom de la chèvre qui constitue à présent près de la moitié du petit bétail (note de l'éditeur).

9. En 1998 sur le grand marché aux bestiaux de Tokmok, une vache laitière s'achetait 10000 soms, un veau d'un mois 1000 à 1200 soms, un cheval environ 8000 soms, un mouton de 1000 à 2500 soms. Et sur le marché d'At Baši, dans la région de Naryn, j'ai relevé les prix suivants la même année : 1600 à 1800 soms pour une vacive (jeune brebis de moins de deux ans), 5300 à 5500 soms pour un poulain, 11000 soms pour une vache laitière, 2000 soms pour un mouton, 10 à 11000 soms pour une jument (alors que le cours du dollar s'établissait alors autour de 47 soms). 
10. Sous la direction du professeur S. G. Kljaštornyj, dans le cadre d'un projet de l'université du Wisconsin mené par A. Hazanov et K. Šapiro.

11. Avec la crise financière, le prix des chevaux de course a baissé ces derniers temps. Certains peuvent s'acheter pour 4000 dollars mais, en moyenne, ils coûtent 15 à 20000 dollars, et parfois bien davantage.

\section{RÉSUMÉS}

L'article examine certains aspects de l'élevage équin au Kirghizstan. Après une rétrospective historique et ethnographique, l'auteur se concentre sur l'aspect actuel de la question. Le rôle économique et culturel du cheval ainsi que les diverses fonctions qu'il remplit sont analysés sur la base de matériaux de terrain. Ce thème permet d'aborder la question des relations entre les autorités locales et la population à l'époque soviétique puis de traiter, entre autres, de la crise de l'élevage dans les années 1990, de l'évolution du cheptel, des particularités de l'utilisation de cet animal à l'époque actuelle, de l'adaptation des éleveurs aux conditions du marché, de la division sexuelle du travail, des soins portés aux chevaux aux différentes saisons, des relations qui s'établissent entre l'homme et le cheval.

In the present paper the author deals with some aspects of horse breeding in Kirghizstan. After a historical and ethnographical retrospective, he focuses on the present situation of Kirghiz horse breeding. He analyses the economic and cultural role of the horse among the Kirghiz on the basis of field research. This allows him to deal with some other questions: power relations between local authorities and the population during the Soviet period, the crisis of animal husbandry in the 1990's, the evolution of livestock, the characteristics of present-day horse utilization, the adaptation of stock breeders to the market economy, gender division of work, the care of horses at different times of the year, relationships between man and horse.

\section{INDEX}

Index géographique : Kirghizstan

Keywords : horse, animal husbandry, privatization, post-soviet transition

Mots-clés : cheval, élevage, privatisation, transition post-soviétique

nomsmotscles Kirghiz

Thèmes : animal, changement, économie, nomadisme

\section{AUTEURS}

\section{AMANTUR ŽAPAROV}

Titulaire d'une thèse de candidat en ethnologie (sur l'élevage traditionnel et l'organisation sociale des Kirghiz), Amantur Žaparov est directeur d'un programme de recherche et d'enseignement à l'Académie des Sciences de la République du Kirghizstan, chercheur associé de 
l'Institut français d'Études sur l'Asie centrale (IFEAC) et coordinateur de son antenne à Bichkek, au Kirghizstan. 